\title{
Analyse de pratiques interdisciplinaires dans l'enseignement supérieur
}

Valérie Camel et Agnès Fargue-Lelièvre

\section{(2) OpenEdition}

Édition électronique

URL : http://journals.openedition.org/ripes/188

DOI : 10.4000/ripes.188

ISSN : 2076-8427

Éditeur

Association internationale de pédagogie universitaire

Référence électronique

Valérie Camel et Agnès Fargue-Lelièvre, "Analyse de pratiques interdisciplinaires dans l'enseignement supérieur », Revue internationale de pédagogie de l'enseignement supérieur [En ligne], 25(2) | 2009, mis en ligne le 14 septembre 2009, consulté le 07 septembre 2020. URL : http://journals.openedition.org/ ripes/188; DOl : https://doi.org/10.4000/ripes.188

Ce document a été généré automatiquement le 7 septembre 2020.

Article L.111-1 du Code de la propriété intellectuelle. 


\title{
Analyse de pratiques interdisciplinaires dans l'enseignement supérieur
}

\author{
Valérie Camel et Agnès Fargue-Lelièvre
}

\section{Introduction}

1 Dans cet article sont présentés et discutés trois exemples de modules interdisciplinaires réalisés depuis plusieurs années à AgroParisTech, établissement d'enseignement supérieur et de recherche. Issu du rapprochement récent de trois établissements (l'Institut national agronomique Paris-Grignon (INA-PG), l'Ecole nationale supérieure des industries agroalimentaires (ENSIA), et l'Ecole nationale du génie rural, des eaux et de la forêt (ENGREF)), AgroParisTech propose actuellement aux étudiants trois cursus distincts: le cursus «ingénieur agronome», le cursus «ingénieur industries agroalimentaires ", et le cursus « ingénieur forestier ». Nous ne mentionnerons ici que des exemples proposés actuellement aux étudiants du cursus « ingénieur agronome », même si d'autres exemples sont également proposés aux étudiants des deux autres cursus.

2 AgroParisTech forme des « ingénieurs du vivant », dont les compétences et les métiers auxquels ils se destinent sont multiples. Leur trait commun est le plus souvent d'aborder une problématique de manière intégrée, en mettant en œuvre des connaissances et des méthodes issues de plusieurs disciplines. C'est la raison pour laquelle la formation de ces ingénieurs par des enseignements interdisciplinaires est cruciale, et ce d'autant plus que le vivant ne peut se concevoir et s'appréhender que par une approche interdisciplinaire. Cette approche peut être transposée à d'autres établissements d'enseignement supérieur. En effet, dans de nombreux domaines, la résolution de problèmes concrets nécessite des approches interdisciplinaires, auxquelles les futurs diplômés doivent donc être formés afin de favoriser leur insertion 
sur le marché de l'emploi (Smidts, Vander Broght, De Kessel, Lebrun \& Schneider, 2004).

3 Au sein d'AgroParisTech l'existence d'enseignements interdisciplinaires dans le cursus «ingénieur agronome» est assez ancienne, mais celle-ci a été formalisée au plan pédagogique en 1994. Il s'agissait à l'époque pour les enseignants de construire des modules longs (deux mois) initiant les étudiants à la gestion de projet par le biais d'une approche de préférence interdisciplinaire. Lors d'une nouvelle réforme pédagogique dans ce cursus il y a cinq ans, l'accent a été mis de nouveau sur la nécessité de former les étudiants par des modules interdisciplinaires, dont la vocation est de permettre aux étudiants d'approfondir des connaissances apportées en tronc commun lors de cours disciplinaires. Cette exigence est réapparue cette année dans le cadre de la réforme engagée au sein de l'établissement sur l'évolution des cursus de formation. L'ensemble des enseignants-chercheurs de l'école s'accorde sur l'importance d'intégrer divers apports disciplinaires autour d'un même objet ou d'une même thématique. Il apparaît en effet crucial que les futurs ingénieurs formés s'approprient la démarche interdisciplinaire pour être à même de résoudre les problèmes complexes auxquels ils seront confrontés dans leur métier futur.

Il est important de préciser ce qu'est l'interdisciplinarité, et en quoi elle se distingue de la pluridisciplinarité, de la multidisciplinarité ou de la transdisciplinarité. En fait, la contribution d'une ou de plusieurs disciplines à l'étude d'un problème ou d'un objet peut se situer sur un continuum, selon le degré d'interaction ou de fusion de ces disciplines, comme suit : monodisciplinarité, pluridisciplinarité ou multidisciplinarité, interdisciplinarité, transdisciplinarité. L'interdisciplinarité suppose un dialogue et l'échange de connaissances, d'analyses, de méthodes entre deux ou plusieurs disciplines ; elle implique qu'il y ait des interactions et un enrichissement mutuel entre plusieurs spécialistes (Etévé \& Champy, 1994). Il s'agit donc de faire interagir plusieurs disciplines (donc plusieurs spécialistes) sur un même objet d'étude. Elle se distingue de la transdisciplinarité, laquelle désigne un savoir qui parcourt plusieurs disciplines sans se soucier de leurs frontières. Elle dépasse la pluridisciplinarité et la multidisciplinarité, qui associent plusieurs disciplines autour d'un thème commun mais dont les contributions respectives sont additionnées, sans réelle interaction entre les disciplines ; la distinction entre pluri- et multi- s'effectue sur les disciplines impliquées, la pluridisciplinarité associant des disciplines très voisines, alors que la multidisciplinarité juxtapose des disciplines parfois très éloignées.

L'interdisciplinarité, en favorisant la recherche des interactions des savoirs et de leur complémentarité, invite à une certaine ouverture d'esprit. Mais il ne s'agit pas d'une valeur en soi, l'interdisciplinarité est avant tout une approche. Celle-ci est au cœur des problématiques curriculaires conçues selon une logique de compétences comme le soulignent Jonnaert, Barrette, Boufrahi \& Masciotra (2004). Sa mise en pratique est diverse, avec un degré plus ou moins poussé d'interconnexion entre les disciplines.

Plusieurs enseignements interdisciplinaires ont été conçus à AgroParisTech, et leur mise en œuvre depuis plusieurs années permet d'avoir un recul sur leur intérêt dans la formation des étudiants, et de dresser un bilan de ces enseignements. Les trois exemples rapportés ci-dessous ont été choisis car ils permettent d'illustrer différents degrés, à travers trois manières possibles de pratiquer l'interdisciplinarité : 1) solliciter différentes disciplines sur un sujet donné ; 2) composer un groupe pluridisciplinaire pour travailler sur un même thème, chacun traitant un aspect particulier ;3) approcher 
globalement un problème puis faire appel aux différentes méthodologies disciplinaires pour analyser la situation et la découper en problèmes et trouver des solutions alternatives (Bailly \& Schils, 1988; Giordan \& Souchon, 1992).

7 Chacun des trois modules présentés ci-dessous sera dans un premier temps décrit en termes d'objectifs pédagogiques, de déroulement et méthodes pédagogiques, et d'évaluation des étudiants. Puis nous effectuerons une analyse de ces pratiques de l'interdisciplinarité, afin de dresser un bilan. Comme nous le verrons par le biais de ces trois exemples, la construction pédagogique d'enseignements interdisciplinaires est parfois délicate, car elle doit s'articuler autour d'un thème cohérent, et surtout permettre une articulation logique et pertinente entre les disciplines impliquées, sans mettre en exergue l'une ou l'autre de ces disciplines au détriment d'une autre. Elle nécessite donc une dynamique concertée entre les enseignants impliqués dans ce type de démarche pédagogique, afin de susciter ensuite l'adhésion des étudiants à cette approche interdisciplinaire.

\section{Présentation des unités d'enseignement interdisciplinaires}

\subsection{Exemple 1 : module d'approfondissement « L'eau : quels traitements pour quelles qualités? »}

8 Le premier exemple concerne un enseignement optionnel dispensé en fin de première année du cursus « ingénieur agronome ». Il s'agit d'un module d'approfondissement de 60 heures, conçu à la fois pour illustrer des connaissances dispensées aux étudiants lors d'enseignements de tronc commun, et leur apporter de nouvelles connaissances. Cet enseignement est organisé par trois départements de l'école : «Sciences et procédés des aliments et bioproduits", "Sciences et ingénierie agronomiques, forestières, de l'eau et de l'environnement » et "Sciences de la vie et santé ». L'équipe pédagogique est bidisciplinaire, constituée de cinq enseignants-chercheurs, dont trois chimistes et deux microbiologistes. Il s'agit donc d'une interdisciplinarité assez limitée, illustration de la pratique « solliciter différentes disciplines sur un sujet donné » (Bailly \& Schils, 1988).

\subsubsection{Présentation générale du module}

L'eau constitue un thème fédérateur, particulièrement propice à la réalisation d'enseignements interdisciplinaires. En effet, l'évaluation de la qualité d'une eau fait appel à des méthodes de chimie analytique et de microbiologie. Quant aux traitements d'une eau pour obtenir une qualité conforme à un usage donné, qu'il s'agisse de traitements de potabilisation ou d'assainissement, ils mettent en œuvre des processus physico-chimiques (ou simplement chimiques) et microbiologiques. Chacun de ces aspects est abordé dans ce module.

\subsubsection{Objectifs pédagogiques}

10 Ce module a été conçu pour permettre aux étudiants de disposer d'éléments de réponse concrets aux questions concernant la qualité et le traitement de l'eau, qui peuvent se poser aujourd'hui aux ingénieurs agronomes. A l'issue de ce module, ils sont ainsi capables de : 1) évaluer la qualité d'une eau en mettant en œuvre certaines méthodes 
d'analyse chimique et bactériologique actuellement utilisées dans les laboratoires de contrôle de la qualité des eaux ; 2) concevoir une filière de traitement de cette eau, en proposant des grands principes de traitement en fonction des objectifs de qualité et des usages de l'eau produite.

\subsubsection{Déroulement et méthodes pédagogiques}

11 Les différents aspects liés à l'eau sont abordés dans ce module : 1) le cycle de l'eau (qualité et quantité) ; 2) la gestion du risque de contamination des eaux ; 3) le contrôle de la qualité des eaux (contextes réglementaires français et européens, échantillonnage, techniques analytiques); 4) les microorganismes des eaux (écologie, épidémiologie) ; 5) les procédés de traitements de l'eau (assainissement, potabilisation: principes, aspects techniques et économiques).

12 Le fil directeur du module est de montrer aux étudiants, d'une part, qu'il existe différents types d'eaux, de qualités diverses, et d'autre part, que l'usage que l'on souhaite faire de cette eau nécessite une qualité particulière, ce qui peut imposer un traitement adapté. Pour ce faire, une partie des connaissances est apportée par des cours et conférences (environ 20 heures) donnés par des enseignants d'AgroParisTech ainsi que par des professionnels du domaine. Quelques cours sont assurés par un duo chimiste-microbiologiste afin de montrer la nécessité d'une approche bidisciplinaire. L'autre apport de connaissances est effectué pendant des séances de travaux pratiques (TP) (environ 20 heures). Dans un premier temps, les étudiants mettent en œuvre les principales méthodes de mesure de la qualité chimique et bactériologique de l'eau ; il s'agit de TP communs, suivis par tous les étudiants du module (cf. figure 1). Dans un deuxième temps, ils se consacrent, sous la forme d'un projet réalisé par binôme, à la détermination des paramètres qui permettent de maitriser les étapes de traitement afin d'obtenir une qualité d'eau en adéquation avec son utilisation. Il s'agit alors de TP de projets, certains concernant un traitement particulier de potabilisation, d'autres un traitement d'assainissement. En lien avec ce projet, les étudiants ont également chacun un article scientifique à analyser. Pour ce faire, des créneaux de travail personnel sont affichés à l'emploi du temps du module, d'autant qu'ils sont également utiles pour permettre aux étudiants d'exploiter leurs résultats de travaux pratiques. Enfin, une demi-journée est consacrée à la visite d'une station de traitement d'assainissement d'eaux résiduaires urbaines. 


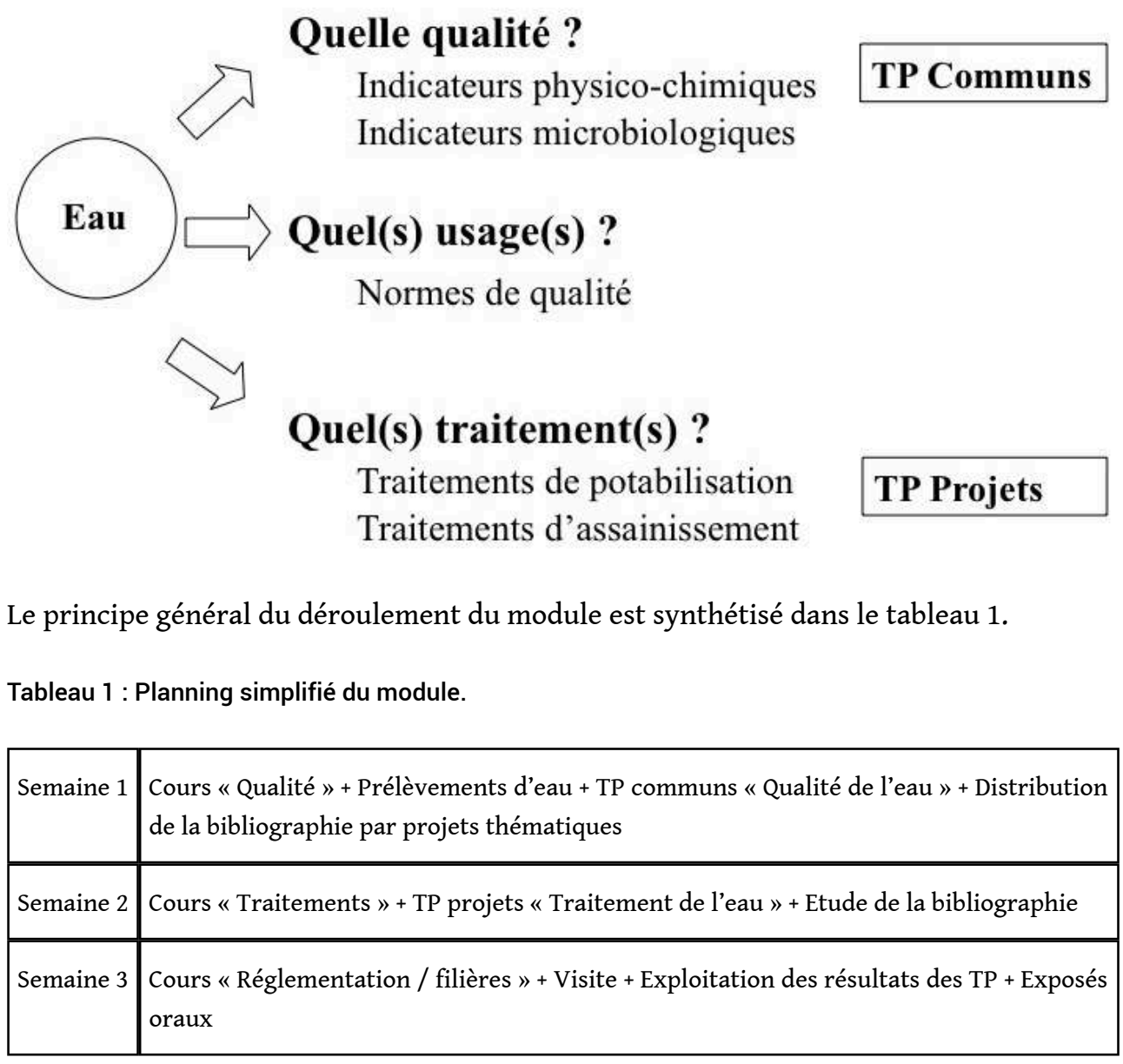

\subsubsection{Evaluation des étudiants}

14 Les étudiants sont évalués sur leurs résultats de travaux pratiques, ainsi que leur analyse d'article. La note finale pour chaque étudiant se décompose comme suit : $25 \%$ pour la synthèse écrite des résultats des TP communs (note du binôme); 25 \% pour le rapport écrit thématique de chaque groupe (note du binôme) ; 25 \% pour l'exposé oral (note de groupe pour les deux ou trois binômes ayant travaillé sur le même thème); $25 \%$ pour le travail fourni individuellement pendant les séances de TP et l'assiduité (note individuelle).

\subsection{Exemple 2 : unité de valeur « Comment analyser les risques alimentaires et assurer la sécurité sanitaire dans les filières agricoles?»}

15 Le second exemple présenté permet d'illustrer la pratique "composer un groupe pluridisciplinaire pour travailler sur un même thème, chacun traitant un aspect particulier " (Bailly \& Schils, 1988). Il s'agit d'une unité de valeur (UV) de 75 heures, proposée de manière optionnelle aux étudiants du cursus « ingénieur agronome » en fin de seconde année. Cet enseignement est organisé par trois départements de l'école : «Sciences et 
procédés des aliments et bioproduits", "Sciences et ingénierie agronomiques, forestières, de l'eau et de l'environnement » et "Sciences de la vie et santé ». L'équipe pédagogique est constituée de quatre enseignants-chercheurs et d'un chercheur, représentant au total quatre disciplines: la zootechnie, l'agronomie, la chimie analytique, l'analyse du risque.

\subsubsection{Présentation générale de l'unité de valeur}

Depuis quelques années on assiste, suite à plusieurs crises (encéphalopathie spongiforme bovine (ESB), dioxine, etc.), à une recrudescence des problèmes de sécurité sanitaire des aliments en Europe. Dans ce contexte, il semble nécessaire d'apporter une meilleure connaissance des relations existant entre les modes de production agricole et la sécurité sanitaire des aliments destinés à l'homme. Pour ce faire, cette UV s'appuie sur des cas d'étude en production végétale et animale, pour mettre l'accent sur les différences entre les filières agroalimentaires du point de vue de la gestion des risques. En effet, alors que les crises sanitaires ont rendu nécessaires des adaptations visibles et sans précédent des différents maillons des filières des produits animaux, les filières végétales, pour l'instant préservées de crises majeures, cherchent à adopter des mesures préventives.

\subsubsection{Objectifs pédagogiques}

17 Cet enseignement a trois objectifs principaux : 1) présenter la démarche d'analyse des risques (évaluation des risques, gestion des risques et communication à propos des risques); 2) faire le point sur les connaissances disponibles et les voies d'action envisageables pour réduire la présence des contaminants en production agricole; 3) identifier les dispositifs et actions mis en œuvre dans les filières pour faire face aux évolutions réglementaires ou aux crises sanitaires. Il permet donc aux étudiants d'appréhender, par une approche interdisciplinaire, les différents aspects de la sécurité sanitaire des aliments, principalement en ce qui concerne les risques chimiques. Ainsi, à l'issue de cette UV, les étudiants sont capables de :1) mettre en œuvre une démarche d'analyse des risques; 2) proposer des actions pour réduire la présence de contaminants en production agricole ; 3) percevoir les dispositifs mis en œuvre dans les filières pour faire face aux évolutions réglementaires ou crises sanitaires.

\subsubsection{Déroulement et méthodes pédagogiques}

Dans cette UV la majeure partie des connaissances est apportée sous forme de cours et conférences donnés par des enseignants-chercheurs d'AgroParisTech, des chercheurs de l'Institut national de la recherche agronomique, ainsi que des intervenants extérieurs. Cela correspond à un volume horaire de 39 heures, réparties entre évaluation du risque (définition-présentation des contaminants en production agricole et des voies de contamination, méthodes d'analyses, construction de la réglementation), gestion du risque (réduction des risques, leviers et dispositifs d'action) et communication autour du risque (perception des risques, certification, médias) (cf. figure 2). 
Figure 2

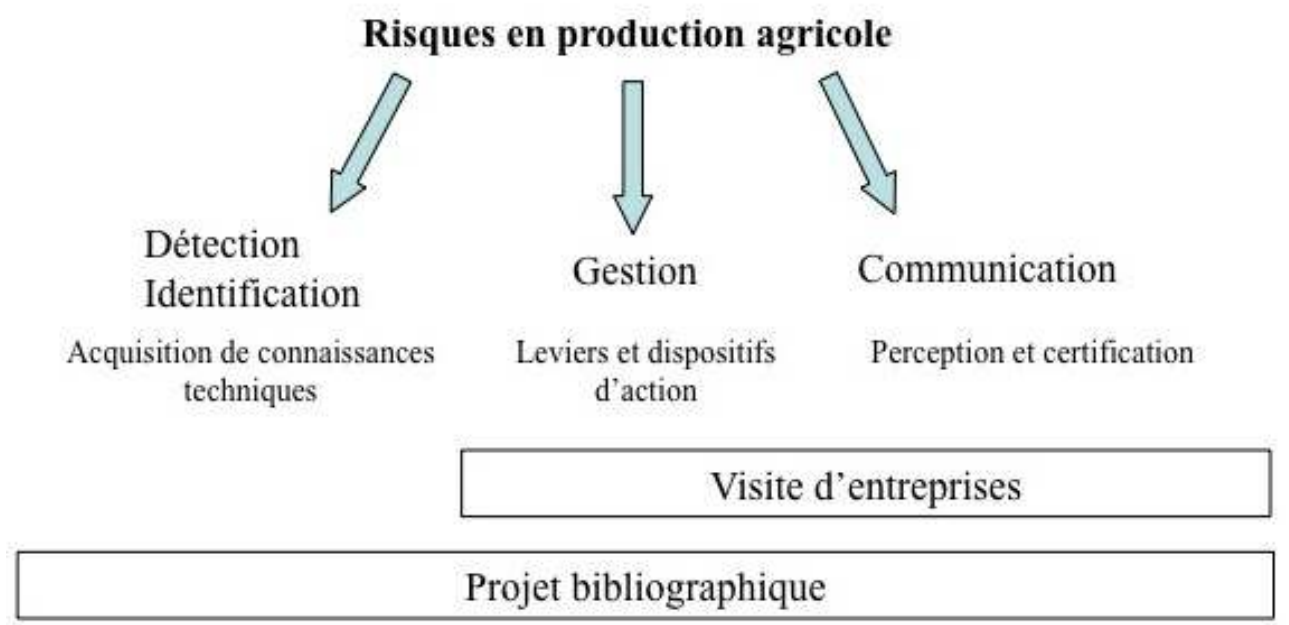

Ces enseignements se basent sur des exemples issus de différents niveaux de filières agricoles et sur différents contaminants (exemples : mycotoxines, acrylamide). Un cours ( 3 heures) bidisciplinaire chimie analytique-technologie alimentaire sur les mycotoxines a été spécialement conçu pour cette UV (cours réalisé en duo par deux enseignants, chacun de l'une des deux disciplines concernées).

Parallèlement, les étudiants ont un projet thématique à mener, par binôme ou trinôme, afin d'étudier la relation entre mode de production et qualité sanitaire. Celui-ci concerne un cas concret de sécurité sanitaire des aliments, qui peut se décliner en termes de produit alimentaire (exemples: poisson, salade) ou de contaminant (exemples : méthylmercure, dioxines). Ce travail est ainsi l'occasion pour les étudiants d'acquérir les connaissances délivrées lors des cours et conférences. Des plages de travail personnel (six demi-journées) sont dégagées à l'emploi du temps pour leur permettre de réaliser leur recherche bibliographique, et d'analyser les documents recueillis (cf. tableau 2). En outre, chaque groupe d'étudiant est encadré par un enseignant de l'équipe pédagogique, qui peut les aider à orienter leur travail si besoin, afin de pouvoir le mener à bien dans le temps imparti.

Tableau 2 : Planning simplifié de l'UV.

\begin{tabular}{|l|l|}
\hline Semaine 1 & $\begin{array}{l}\text { Cours (présentation des contaminants, évaluation et maîtrise des risques en } \\
\text { production agricole) + Distribution de la bibliographie par projet thématique }\end{array}$ \\
\hline Semaine 2 & Cours (gestion et perception du risque) + Travail personnel \\
\hline Semaine 3 & Visites (2 entreprises) + Travail personnel \\
\hline Semaine 4 & Cours (gestion et perception du risque) + Travail personnel \\
\hline $\begin{array}{l}\text { Semaine } 5 \\
(1 / 2 \text { semaine) }\end{array}$ & Travail personnel + exposés oraux par projet thématique \\
\hline
\end{tabular}


21 végétal, présentant chacune un ou des risque(s) de contamination et les choix de
gestion de ce(s) risque(s) au sein de l'entreprise ou de la filière.

\subsubsection{Evaluation des étudiants}

L'évaluation porte sur le projet thématique réalisé qu'ils restituent sous forme d'exposé oral ainsi que par la rédaction d'un rapport écrit. Par la suite, la note finale pour chaque étudiant est calculée avec la pondération suivante : $50 \%$ pour le contenu et la forme du rapport de chaque groupe (note de groupe) ; 50 \% pour la qualité de l'exposé oral de chaque groupe (note de groupe).

\subsection{Exemple 3 : initiation à l'ingénierie de projet « Gestion des eaux dans un bassin versant »}

Le troisième exemple présenté concerne également un enseignement proposé aux étudiants de deuxième année du cursus «ingénieur agronome». Il s'agit d'un enseignement optionnel de 150 heures, intitulé "Gestion des eaux dans un bassin versant ", proposé dans le cadre de ce que l'on appelle les Initiations à l'Ingénierie de Projet (INIP). Il est proposé conjointement par trois départements d'AgroParisTech : "Sciences et procédés des aliments et bioproduits", "Sciences et ingénierie agronomiques, forestières, de l'eau et de l'environnement » et « Sciences économiques, sociales et de gestion ". L'équipe pédagogique est constituée de six enseignantschercheurs, représentant au total cinq disciplines : l'agronomie, la pédologie, la chimie analytique, la sociologie et le droit. La conception de cet enseignement permet d'illustrer la pratique « approcher globalement un problème puis faire appel aux différentes méthodologies disciplinaires pour analyser la situation et la découper en problèmes et trouver des solutions alternatives (p.139)» (Giordan \& Souchon, 1992).

\subsubsection{Présentation générale de l'initiation à l'ingénierie de projet}

Cet enseignement interdisciplinaire est construit autour de la problématique de la gestion des eaux dans un bassin versant (Camel \& Martin, 2003). En effet, la gestion de l'eau, ou plus justement des eaux (souterraines et superficielles), est une préoccupation essentielle des ingénieurs du $21^{\text {ème }}$ siècle. Il s'agit de prendre en compte une ressource tantôt limitée, tantôt excédentaire, destinée à des usages multiples, avec une demande croissante en qualité. Ce bien doit donc être géré dans le temps et dans l'espace avec un souci et une volonté d'intégration des contraintes économiques et environnementales. Cette gestion des eaux passe notamment par une évaluation des risques tant d'un point de vue qualitatif (contamination) que quantitatif (sécheresse, inondation) et la mise en oeuvre de mesures curatives et/ou de prévention.

\subsubsection{Objectifs pédagogiques}

A l'issue de cette INIP, les étudiants sont capables de : 1) recueillir auprès des différents acteurs l'information nécessaire à la conduite de leur projet ; 2) faire l'analyse et la synthèse de documents divers (rapport d'études, photos aériennes, données pluviométriques, résultats d'analyses des eaux, etc.) ; 3) s'organiser pour travailler en

Revue internationale de pédagogie de l'enseignement supérieur, 25(2) | 2009 
groupe sur des projets thématiques concrets ; 4) proposer des actions hiérarchisées à l'échelle d'un bassin versant, en intégrant les points de vue des différents acteurs concernés par la gestion des eaux; 5) valoriser leur travail par la production de supports de qualité (schémas, diaporamas).

\subsubsection{Déroulement et méthodes pédagogiques}

L'INIP se situe dans un espace limité à un bassin versant. Cette entité géographique permet de prendre en considération l'état, la circulation, l'utilisation des eaux et des zones humides, en identifiant les conséquences écologiques, agronomiques et socioéconomiques. Une telle démarche s'insère dans le cadre d'une politique régionale ou départementale qui demande parfois à être clarifiée.

Une analyse interdisciplinaire conduit à établir un état des lieux du bassin versant (exemple: occupation du territoire par des activités agricoles, industrielles ou récréatives, et conséquences sur la circulation et la qualité des eaux) et à approfondir certains thèmes pour répondre aux préoccupations des acteurs concernés (cf. figure 3 ). Ce travail a pour but d'aider à la décision par des propositions d'action cohérentes pour l'usage et la gestion des eaux du territoire étudié (prise en compte des différents impératifs de qualité, quantité, réglementation, vie économique et sociale, etc.).

Figure 3

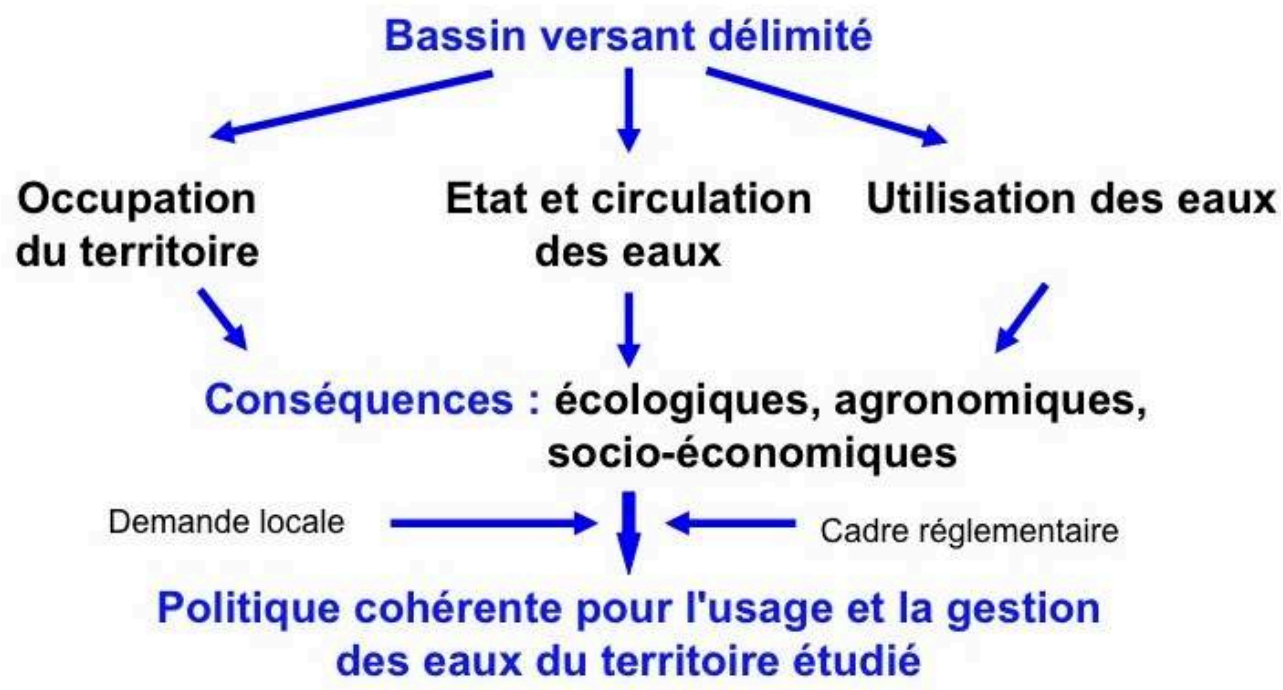

L'INIP se déroule en trois périodes (cf. tableau 3) qui correspondent aux phases de déroulement de tout projet d'ingénieur agronome.

Tableau 3 : Planning simplifié de l'INIP.

\begin{tabular}{|l|l|l|}
\hline Phase 1 & Semaine 1 & Voyage + Ateliers + Cours \\
\hline \hline & Semaine 2 & $\begin{array}{l}\text { Ateliers + Cours + Présentation des ateliers + rédaction des rapports d'ateliers } \\
+ \text { répartition par thèmes }\end{array}$ \\
\hline & Semaine 3 & Cours + Organisation terrain \\
\hline
\end{tabular}




\begin{tabular}{|l|l|l|}
\hline Phase 2 & Semaine 4 & Terrain \\
\hline & Semaine 5 & Terrain \\
\hline & Semaine 6 & Présentation des thèmes + rédaction des rapports thématiques \\
\hline Phase 3 & Semaine 7 & Préparation de l'oral final + correction des rapports thématiques \\
\hline & Semaine 8 & Présentation orale finale + rédaction des rapports thématiques \\
\hline
\end{tabular}

29 Phase 1 - Découverte du terrain et des acteurs; définition de la problématique (trois semaines)

Cette période se décline en trois phases: 1) rencontre avec les partenaires locaux, intégration des différents points de vue et visite de la zone d'étude (voyage de deux à trois jours) ; 2) lors de quatre séances d'ateliers, analyse sur documents de la zone d'étude (photos aériennes, recensements, etc.) et première élaboration des problématiques ; 3) traduction des questions des acteurs en projets thématiques. Un apport de connaissances adaptées aux besoins des thèmes abordés est proposé aux étudiants par les enseignants des trois départements d'AgroParisTech impliqués dans cet enseignement.

\section{Phase 2 - Réalisation des projets thématiques (trois semaines)}

Chaque groupe (deux à quatre étudiants) s'approprie un thème d'étude pour lequel il établit un diagnostic et formule des propositions d'actions. Pour ce faire, les étudiants vont sur le terrain rencontrer les acteurs locaux et récolter les informations nécessaires à la réalisation de leur projet. Ce travail donne lieu à une restitution écrite et orale par groupe, devant les enseignants et les étudiants.

31 Phase 3 - Synthèse et restitution finale (deux semaines)

Dans cette dernière phase du projet, les étudiants mettent en commun leurs réflexions afin de produire un ensemble cohérent de diagnostics et de propositions d'actions. Ceci donne lieu à une présentation orale devant les partenaires locaux, qui sont amenés à réagir devant les résultats de l'étude. L'ensemble (rapports écrits et supports de présentation orale) est ensuite compilé sous format électronique afin de le diffuser aux acteurs locaux et régionaux.

32 Chaque année, le bassin versant est choisi en concertation avec des décideurs locaux. Par le passé les bassins versants de l'Yvette (Essonne), de la Maye, la Vimeuse et la Cologne (Somme), de la Lieure et du Fouillebroc (Eure), de la Vienne et l'Austreberthe (Seine-Maritime), du Chéran (Mayenne), du Morbras (Val-de-Marne et Seine-et-Marne), de l'Arré (Oise), de la Nièvre d'Arzembouy et de la Vrille (Nièvre et Yonne) ont été étudiés. A titre d'exemple, sur la Nièvre, les étudiants ont travaillé sur les problématiques suivantes : état des lieux des ouvrages hydrauliques et de leur gestion, impact des pratiques agricoles sur la qualité de l'eau d'un captage, gestion de l'assainissement, choix de gestion collective de l'eau (SIAFEE, 2007). 


\subsubsection{Evaluation des étudiants} $2 / 3$ sur leur travail de thème (le barème correspond au temps imparti à chacune de ces activités). Pour chacune de ces activités (atelier et thème), les étudiants sont notés ensuite avec la pondération suivante : $1 / 3$ pour le contenu et la forme du rapport écrit (note de groupe, d'atelier ou de thème selon le cas) ; $1 / 3$ pour la qualité des exposés oraux de chaque groupe (note de groupe, d'atelier ou de thème) ; $1 / 3$ pour le travail fourni individuellement pendant les séances de travail collectif et sur le terrain (note individuelle)

\section{Evaluation et discussion}

Ces trois exemples d'enseignements interdisciplinaires sont mis en œuvre à AgroParisTech depuis plusieurs années (cinq ans pour le module, trois ans pour l'UV et quinze ans pour l'INIP). Il est donc possible d'en tirer un bilan, à la fois du point de vue des étudiants que de celui des enseignants.

\subsection{La perception des étudiants}

\subsubsection{Quelles modalités d'évaluation de ces enseignements ?}

L'évaluation faite par les étudiants est conduite de deux manières. En premier lieu, une grille d'évaluation type (mais adaptée bien entendu à l'unité d'enseignement évaluée) leur est distribuée durant les premiers jours de l'enseignement (cf. figure 4). Ainsi, ils remplissent la grille au fil du déroulement de l'enseignement, et ils la remettent aux enseignants à la fin du module (soit individuellement, soit de manière collective pour respecter leur anonymat). En second lieu, le dernier jour de l'unité d'enseignement est effectué un bilan oral collectif, animé par les enseignants. L'expérience montre que ces deux bilans, s'ils sont globalement concordants, offrent également une certaine complémentarité. En effet, le taux de retour des grilles remplies n'est jamais de $100 \%$ (il peut osciller entre 50 et $90 \%$ généralement) ; par conséquent le bilan oral est l'occasion, pour les étudiants n'ayant pas souhaité ou pris le temps de remplir cette grille, d'exprimer leur point de vue. De la même manière, un bilan oral ne suffit pas, car il subsiste toujours quelques étudiants qui n'osent pas s'exprimer (d'autant plus que l'évaluation ne peut plus être anonyme). De surcroît, l'utilisation de la grille d'évaluation permet d'avoir une certaine quantification de la satisfaction des étudiants sur certains items, et donc d'effectuer ensuite des tendances sur la population d'étudiants ayant suivi l'enseignement en question. Ainsi, cela est utile pour faire évoluer les enseignements d'année en année, en les améliorant. 
Figure 4

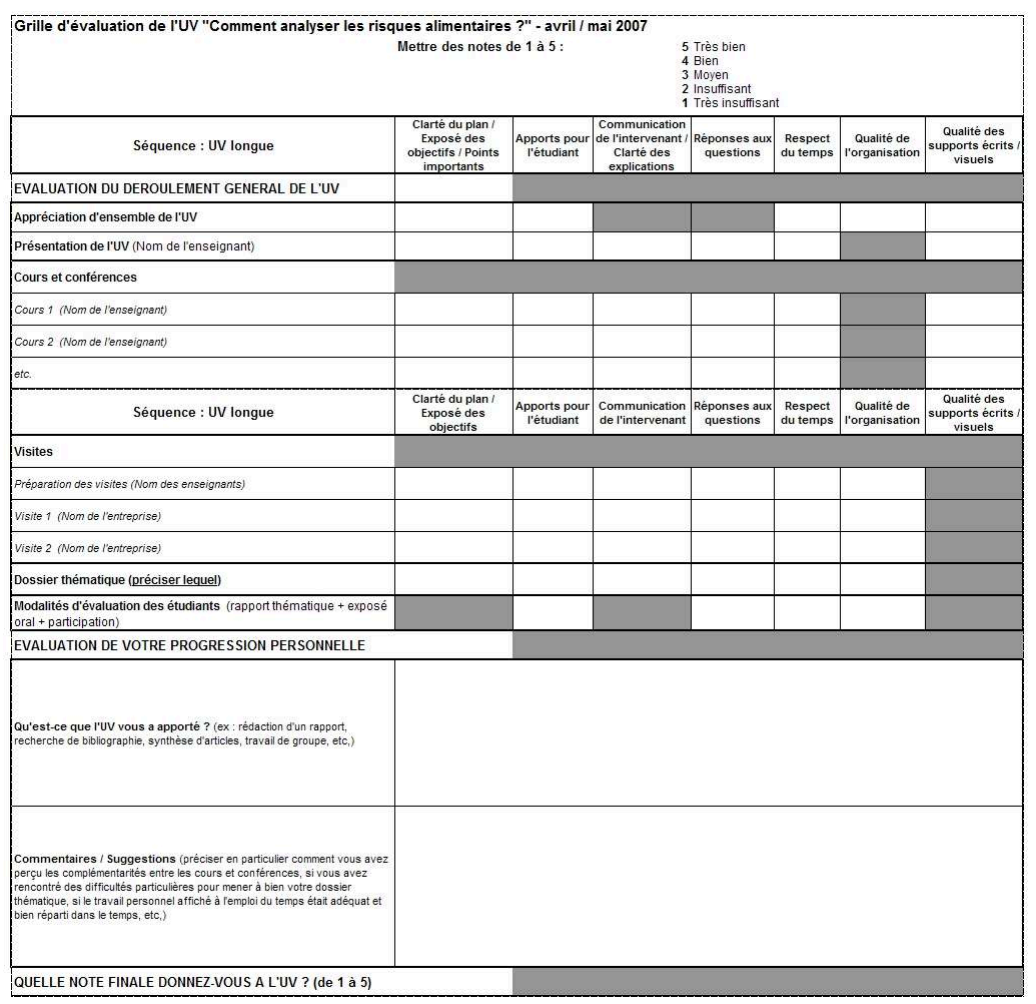

Depuis 2002, l'utilisation conjointe du questionnaire et du bilan oral a permis de recueillir l'avis d'environ $80 \%$ des élèves de ces trois modules pour un total de 40 à 60 étudiants par an (en moyenne 20 pour le module sur l'eau, 5-15 pour le module sur la sécurité sanitaire et 15-25 pour le module d'initiation à l'ingénierie de projet).

\subsubsection{Comment les étudiants perçoivent-ils ces enseignements?}

Les étudiants sont le plus souvent demandeurs de ce type d'enseignement, car il leur offre une approche intégrée d'un sujet d'étude donné. Et de fait, en pratique, ils sont dans leur grande majorité conquis par ces enseignements interdisciplinaires. L'intégration de différentes manières d'appréhender un même objet, et de plusieurs méthodes pour étudier cet objet, leur permet de saisir la complexité du réel, tout en acquérant des raisonnements et des méthodes disciplinaires. L'approche interdisciplinaire qu'ils expérimentent lors de travaux de groupe (qu'il s'agisse de travaux pratiques, de travaux dirigés, ou de projets sur le terrain) est l'occasion pour eux d'établir des liens ou des interactions entre des disciplines habituellement traitées séparément. En outre, ils apprécient la diversité des enseignants qui les encadrent, tant d'un point de vue disciplinaire qu'humain.

Toutefois, les étudiants sont encore peu familiers avec ce type d'enseignement, surtout lorsqu'ils sont en première année d'école puisqu'ils sont issus pour la plupart de classes préparatoires scientifiques, dont la formation a jusqu'ici été principalement conçue comme un apport de connaissances disciplinaires. Certains d'entre eux ont donc du mal à concevoir que les enseignants puissent avoir des visions ou des approches différentes pour un même objet ou une même thématique. Le fait de prendre conscience qu'il y a plusieurs manières d'appréhender le réel, et par suite plusieurs solutions ou plusieurs "vérités ", peut les dérouter. Quelques étudiants ont alors tendance à se focaliser sur 
une discipline, d'où l'importance d'avoir des enseignants d'autres disciplines pour les aider à ne pas négliger certains aspects importants des objets étudiés.

L'expérience acquise au fil de ces années montre que l'auto-apprentissage constitue un passage important, voire nécessaire, pour permettre aux étudiants de prendre du recul et de mûrir leur réflexion, afin d'être en mesure de s'approprier cette approche interdisciplinaire. Par la suite, des créneaux dédiés au travail personnel ont été introduits dans l'emploi du temps des trois modules décrits, avec un volume horaire qui a sensiblement augmenté par rapport aux premières années de mise en œuvre de ces enseignements; il est d'autant plus important que le degré d'interdisciplinarité est élevé dans le module. En effet, comme le souligne Rege Colet (2002), «les théories de l'apprentissage dans le cadre de l'enseignement interdisciplinaire mettent l'accent sur l'autonomie de l'apprenant et sur l'acquisition d'une méthode de travail ou d'un raisonnement critique (p.78)». Ce point important de la pédagogie a d'ailleurs fait l'objet de discussions souvent animées au sein des équipes pédagogiques concernées, les enseignants ayant souvent de par leur expérience disciplinaire des regards assez divergents sur l'utilité de cet auto-apprentissage. L'ajustement d'un temps adapté de travail personnel s'est fait par l'expérience, au fil des années. Nous rejoignons ici le constat d'Asloum et Bouillier (2007) qui notent que l'engagement de l'équipe pédagogique dans une logique d'essais et d'erreurs est fondamental lorsqu'il s'agit d'enseignements interdisciplinaires.

La mise en situation des étudiants lors de travaux de groupe apparaît également comme un volet important pour leur apprentissage de l'interdisciplinarité, le travail en groupe étant pour eux l'occasion de confronter différents points de vue, de développer leur sens critique et leur faculté de synthèse tout en acceptant le débat pour faire émerger un consensus au sein du groupe. En contrepartie, il en découle pour les enseignants une difficulté dans l'évaluation individuelle du travail des étudiants.

\subsection{L'analyse des enseignants}

\subsubsection{Quelle approche de l'interdisciplinarité ?}

Dans les trois exemples présentés, la démarche interdisciplinaire choisie peut être considérée comme une approche "instrumentale» dont la finalité est la résolution d'un problème concret, et où "l'interdisciplinarité est perçue comme une pratique essentiellement politique, c'est-à-dire comme une négociation entre différents points de vue pour finalement décider d'une représentation considérée comme adéquate en vue d'une action (p. 139)» (Fourez, 2001). Ces exemples permettent d'illustrer la possibilité d'introduire de l'interdisciplinarité modulable dans les enseignements, en lien notamment avec les volumes horaires alloués à ces enseignements. Ainsi, dans le module sur l'eau, il s'agit davantage d'une approche pluridisciplinaire, l'interdisciplinarité étant mise en avant principalement lors de cours ou du traitement des résultats d'analyse pour évaluer la qualité de l'eau. Dans l'UV sur les risques sanitaires, le caractère interdisciplinaire est plus marqué, car cette approche est nécessaire pour appréhender l'ensemble d'une filière agroalimentaire. Enfin, dans l'INIP sur la gestion des eaux, l'interdisciplinarité est très forte, avec une parfaite illustration d'une « interdisciplinarité de projet, centrée sur la pratique et articulée sur le terrain (p.134)» (Lenoir et Sauvé, 1998). Dans ces trois exemples, on peut dire qu'il s'agit typiquement d'une interdisciplinarité restrictive au sens donné par Boisot (1971) : « les interactions mises en jeu par les disciplines en présence 
n'intéressent que leurs interfaces. Il n'y a plus osmose réciproque des éléments constitutifs des disciplines - notamment des lois - et par conséquent les deux formes d'intégration précédemment proposées [fécondation de plusieurs disciplines par les mêmes lois interdisciplinarité linéaire- et engendrer une discipline nouvelle - interdisciplinarité structurale] sont en principe absentes. Les influences mutuelles s'exercent par l'établissement de contraintes, par le fait que chaque discipline impliquée proposera, pour son propre compte, des limites au champ des autres (....) ces frontières découpent dans le réel une zone restreinte, interdisant aux interdisciplinaires de se situer à son extérieur. Cette zone est la zone du possible, celle qui comprend quelque part la situation optimalisée en fonction des données et des contraintes, par l'ajustement des facteurs qui la déterminent (p.33-34).»

\subsubsection{Comment mettre en oeuvre cette interdisciplinarité ?}

Des réunions pédagogiques réalisées en début et fin de chacun des trois modules permettent à l'équipe pédagogique de dresser un bilan de l'année précédente et de préparer l'année à venir en apportant les améliorations nécessaires à un bon déroulement de ces enseignements. En pratique, plus le caractère interdisciplinaire d'un enseignement est marqué, plus l'implication de l'équipe pédagogique doit être forte. En outre, il est important de construire des enseignements autour d'un thème cohérent, afin de permettre une articulation logique et pertinente entre les disciplines impliquées, sans mettre en exergue l'une ou l'autre de ces disciplines au détriment d'une autre. En effet "l'interdisciplinarité vise à terme une synthèse et il importe donc d'organiser des situations d'enseignement et d'apprentissage où cette synthèse peut se construire (p.69) ( (Rege Colet, 2002).Une dynamique concertée entre les enseignants impliqués dans une telle démarche pédagogique est donc cruciale dans ce type d'enseignement. Aussi est-il nécessaire que chaque enseignant prenne conscience des références de sa propre discipline, afin d'être en mesure de travailler au sein d'une équipe pédagogique. Il faut également que chaque enseignant accepte de ne pas maitriser tout le contenu de l'enseignement aux yeux des étudiants. Or, il est souvent difficile pour un enseignant de savoir "comment se situer face aux élèves, sans maîtriser suffisamment le contenu (p.2)» comme le souligne Partoune (2008). Cette dernière met en exergue le fait que « la remise en question du statut de l'enseignant dans le cadre d'un apprentissage ouvert est au cour de l'anxiété ressentie (p.2)» souvent par l'enseignant. Dans notre pratique de l'interdisciplinarité dans les trois modules décrits, cette difficulté personnelle semble avoir été surmontée par tous les enseignants. Nous pensons que ceci résulte, d'une part, de l'organisation de réunions régulières entre les étudiants et l'ensemble de l'équipe enseignante pour faire le point sur l'avancement du travail des étudiants et leurs questionnements, et d'autre part, d'un réel travail d'équipe des enseignants (l'appartenance à une équipe aide l'enseignant à asseoir sa situation face aux élèves).

Par conséquent, le développement d'enseignements interdisciplinaires reste en pratique plus complexe qu'un enseignement pluridisciplinaire, et a fortiori monodisciplinaire. La conception de tels enseignements doit donc être discutée au préalable entre les enseignants concernés, et leur réalisation requiert l'adhésion totale de ces enseignants. Or, cette dernière n'est pas toujours facile à atteindre, les enseignants ayant été initialement formés par une approche souvent essentiellement disciplinaire. Dans nos modules, il aura fallu environ quatre à cinq ans pour que les enseignements arrivent à une certaine stabilité dans leur construction en fonction de la complexité du module. En outre, se pose alors le problème de la gestion de cette 
interdisciplinarité, qui peut s'avérer délicate. Ainsi, comme le soulignent Lenoir et Sauvé (1998), « ce sont sans doute les problèmes organisationnels qui constituent les principaux obstacles au recours à l'interdisciplinarité (p.141) ». Dans nos expériences pratiques, les groupes d'enseignants impliqués se sont auto-organisés, sans qu'il y ait de position dominante clairement affirmée pour l'un ou l'autre des enseignants. Il en résulte de fait une responsabilité pédagogique des modules partagée entre plusieurs enseignants, ce qui implique un réel travail d'équipe. La question est de savoir si c'est l'interdisciplinarité qui favorise l'auto-organisation ou si c'est au contraire l'autoorganisation qui permet à l'interdisciplinarité de s'exprimer (Sandoz-Guermond, Benmohammed, Boutros-Saikaili, Filippi, Forest, George, Leleve \& Michel, 2004). A la lumière de nos années de pratique, nous pensons que l'auto-organisation constitue l'un des piliers de la viabilité de ces enseignements, plaçant ainsi les enseignants sur un pied d'égalité sans distinction de leur discipline, et dans une position d'écoute de l'autre, favorisant ainsi leur volonté de travailler en équipe.

\subsubsection{Quels intérêts pour les enseignants ?} exemple, l'articulation de certains cours disciplinaires a conduit depuis quelques années à s'orienter vers la création de cours bidisciplinaires dans ces modules, afin de présenter aux étudiants la complémentarité et l'interconnexion entre deux disciplines : un cours sur l'assainissement des eaux usées (chimie-microbiologie), et un cours sur les mycotoxines (chimie-technologie alimentaire). Outre l'intérêt pour les étudiants (cours plus dynamique, compléments ou autre point de vue apportés par chaque enseignant sur les différentes parties du cours ce qui permet souvent une meilleure 
compréhension ou une mise en évidence des points clés), ce type de pratique pédagogique sert aussi les enseignements, chacun étant ainsi amené à porter un autre regard sur l'objet ou le thème enseigné, et de ce fait à modifier l'enseignement de sa propre discipline. Un autre exemple concerne la conduite de travaux de groupes : cette pratique peut être fort différente d'une discipline à l'autre, d'où un enrichissement mutuel des enseignants au travers d'une gestion pédagogique concertée.

\section{Conclusion}

Les exemples d'enseignement présentés, et expérimentés depuis plusieurs années au sein d'AgroParisTech, illustrent différentes mises en œuvre possibles de l'interdisciplinarité, avec des degrés variables. Si la construction et la réalisation de tels enseignements ne vont pas de soi, et nécessitent une implication pédagogique forte des enseignants, les atouts qu'ils constituent dans la formation des étudiants justifient les efforts nécessaires à leur réalisation. Il convient en particulier que les enseignants aient une réflexion concertée sur la manière de pratiquer cette interdisciplinarité, et que ceci soit réalisé autour d'un thème fédérateur cohérent. Il faut aussi souligner la nécessité d'inclure dans les pratiques pédagogiques l'auto-apprentissage, étape nécessaire pour permettre aux étudiants de s'approprier cette interdisciplinarité.

Au sein d'AgroParisTech une réforme des cursus de formation est engagée actuellement pour construire un nouveau cursus ingénieur, sur la base des trois cursus préexistants des trois écoles fondatrices d'AgroParisTech. Dans ce cadre, les compétences des futurs diplômés ont été identifiées, en vue d'élaborer le programme de formation dans une logique de compétences. Il s'ensuite une demande forte, émanant à la fois de la direction et des enseignants, d'introduire dans la formation curriculaire des séquences d'apprentissage favorisant pour l'apprenant l'intégration de plusieurs savoirs disciplinaires. Ainsi, l'interdisciplinarité sera une composante essentielle des enseignements du nouveau cursus ingénieur de cette école. Les expériences menées auparavant dans ce domaine, tels que les trois modules décrits dans cet article, seront donc utiles pour bâtir de nouveaux enseignements interdisciplinaires. Toutefois, il nous semble important que la construction de tels enseignements soit laissée à l'initiative des enseignants, car l'interdisciplinarité ne pourra s'exprimer en pratique que si les enseignants mobilisés adhèrent pleinement à cette approche, afin d'adopter une posture qui leur permette de s'ouvrir aux autres disciplines tout en acceptant de ne pas maîtriser tout le contenu de l'enseignement.

\section{BIBLIOGRAPHIE}

Asloum, N., \& Bouillier, M. H. (2007, août). Pratiques collectives d'enseignants engagés dans une formation interdisciplinaire. Communication présentée au colloque « Actualités de la Recherche en Education et en Formation », Strasbourg, France. 
Bailly, J.-M., \& Schils, J. (1988, janvier). Trois niveaux d'interdisciplinarité dans l'enseignement secondaire. Communication présentée à la journée pédagogique FNEC, Bruxelles, Belgique.

Boisot, M.H. (1971). Discipline, interdisciplinarité, programme interdisciplinaire. Revue Française de Pédagogie, 17, 32-38.

Camel, V., \& Martin, P. (2003, juin). Une approche pluridisciplinaire de la pédagogie par projet: la gestion des eaux dans un bassin versant. Communication présentée au 2e colloque «Questions de pédagogie dans l'enseignement supérieur: réflexion, projets et pratiques », Brest, France.

Duchastel, J., \& Laberge, D. (1999). La recherche comme espace de médiation interdisciplinaire. Sociologie et sociétés, 31, 63-76.

Etévé, C., \& Champy, P. (1994). Dictionnaire encyclopédique de l'enseignement et de la formation. Paris : Nathan.

Fourez, G. (2001). La construction des sciences. Les logiques des inventions scientifiques : introduction à la philosophie et à l'éthique des sciences. Bruxelles : de Boeck Université.

Giordan, A., \& Souchon, C. (1992). Une éducation pour l'environnement. Paris : Z'Editions.

Jonnaert, P., Barrette, J., Boufrahi, S., \& Masciotra, D. (2004). Contribution critique au développement des programmes d'études : compétences, constructivisme et interdisciplinarité. Revue des sciences de l'éducation, 30, 667-696.

Lenoir, Y., \& Sauvé, L. (1998). De l'interdisciplinarité scolaire à l'interdisciplinarité dans la formation à l'enseignement: un état de la question. 1 - Nécessité de l'interdisciplinaire et rappel historique. Revue Française de Pédagogie, 124, 121-153.

Partoune, C. (2008). Interdisciplinarité. Consulté le 28 janvier 2009 à : http://www.lmg.ulg.ac.be/ articles/interdisciplinarite/index.htm

Rege Colet, N. (2002). Enseignement universitaire et interdisciplinarité - un cadre pour analyser, agir et évaluer. Bruxelles : De Boeck.

Sandoz-Guermond, F., Benmohamed, H., Boutros-Saikaili, N., Filippi, M., Forest, J., George, S., Leleve, A., \& Michel, C. (2004, septembre). Interdisciplinarité STIC-SHS : l'expérience du groupe CESTIC. Communication présentée au colloque «A la croisée des Sciences, pratiquer et comprendre l'interdisciplinarité », Aix-en-Provence, France.

SIAFEE (Sciences et ingénierie agronomiques, forestières, de l'eau et de l'environnement) (2007). INIP-BV-2006-07. Consulté le 21 juin 2009 à : http://www.siafee-agroparistech.fr/enseignements/ inip-bv/inip-bv-2006-07

Smidts, D., Vander Broght, C., De Kesel, M., Lebrun, M., \& Schneider, Y.-J. (2004, mai). L'apprentissage interdisciplinaire en Sciences de la Vie : faciliter l'intégration des contenus. Communication présentée au $21^{\text {ème }}$ congrès de l'AIPU, Marrakech, Maroc.

\section{RÉSUMÉS}

Cette étude dresse le bilan de plusieurs années de mise en oeuvre de trois modules d'enseignement avec différents degrés d'interdisciplinarité dans une formation de second cycle d'élèves-ingénieurs. Elle examine à la fois les questions d'organisation et de pédagogie, mais également les bénéfices et contraintes pour les étudiants et les enseignants. Ces enseignements sont appréciés des étudiants, car ils leur permettent d'établir des liens ou des interactions entre des disciplines habituellement traitées séparément, même s'ils sont parfois un peu 
décontenancés par cette approche qui diffère de l'approche monodisciplinaire à laquelle ils sont habitués. L'auto-apprentissage s'avère essentiel pour favoriser l'intégration des savoirs disciplinaires par les apprenants. Quant aux enseignants, malgré un investissement plus important, ils y trouvent un intérêt certain, avec en particulier une ouverture qu'ils peuvent mettre à profit dans leurs autres enseignements et leur pratique de la recherche. En effet, adopter un point de vue interdisciplinaire permet de porter un nouveau regard sur sa discipline.

This study is a report covering several years on three teaching modules with different degrees of interdisciplinarity for engineering students. It examines issues regarding organisation and pedagogy and also advantages and limits both for students and teachers. These teaching modules are well appreciated by students, as they offer them an opportunity to establish links or interactions between disciplines usually dealt with separately, even though they may be somewhat disconcerted by this approach which differs from the monodisciplinary approach that they are familiar with. Self-learning is essential in order to encourage integration of disciplinary knowledge by the learners. As for the teachers, despite a higher investment requested for developing these interdisciplinary modules, they find a real interest, especially as far as opening up to other disciplines, that they can use in other teaching modules and in their research practice. Indeed, adopting an interdisciplinary approach offers a new perspective on one's own discipline.

\section{INDEX}

Mots-clés : enseignement supérieur, évaluation, interdisciplinarité, pédagogie, pratiques

Keywords : evaluation, higher education, interdisciplinarity, pedagogy, practice

\section{AUTEURS}

\section{VALÉRIE CAMEL}

Maître de conférence

UFR Chimie analytique

AgroParisTech

Département Sciences et procédés des aliments et bioproduits

16 rue Claude Bernard

F-75005 Paris

Courriel : valerie.camel@agroparistech.fr

\section{AGNÈS FARGUE-LELIÈVRE}

Maître de conférence

UFR Décision, exploitations, filières

AgroParisTech

Département Sciences et ingénierie agronomiques, forestières, de l'eau et de l'environnement

16 rue Claude Bernard

F-75005 Paris

Courriel : agnes.lelievre@agroparistech.fr 